\title{
Tomate (Solanum lycopersicum L.) cultivado bajo invernadero: correlaciones entre variables
} Tomato (Solanum lycopersicum L.) grown under greenhouse conditions: correlations among variables

\author{
José Eladio Monge-Pérez ${ }^{1}$, Michelle Loría-Coto²
}

Fecha de recepción: 31 de octubre de 2018

Fecha de aceptación: 18 de diciembre de 2018

Monge-Pérez, J; Loría-Coto, M. Tomate (Solanum lycoper-

sicum L.) cultivado bajo invernadero: correlaciones entre variables. Tecnología en Marcha. Vol. 32-3. Julio-Setiembre

2019. Pág. 37-54.

DOI: https://doi.org/10.18845/tm.v32i3.4478

1 Docente en la Sede de Guanacaste, e Investigador en la Estación Experimental Agrícola Fabio Baudrit Moreno, Universidad de Costa Rica. Costa Rica. Correo electrónico: melonescr@yahoo.com.mx. (iD) https://orcid.org/0000-0002-5384-507X

2 Investigadora y docente, Escuela de Ciencias Exactas y Naturales, Universidad Estatal a Distancia, Costa Rica. Correo electrónico: michelle_loria@yahoo.com. (i) https://orcid.org/0000-0003-0456-2230 


\title{
Palabras clave
}

Solanum lycopersicum; calidad; rendimiento; 'Brix; correlación de Pearson; regresión lineal.

\section{Resumen}

Se establecieron correlaciones de Pearson para 63 genotipos de tomate producidos bajo invernadero, entre siete variables cuantitativas: edad al inicio de la cosecha (ddt), peso del fruto (g), número de frutos por racimo, rendimiento (ton/ha), firmeza del fruto $(\mathrm{N})$, porcentaje de sólidos solubles totales ( ${ }^{\circ} \mathrm{Brix}$ ), y $\mathrm{pH}$ del jugo del fruto. Las únicas correlaciones de Pearson evaluadas en los 63 genotipos que fueron altas $(r \geq 0,69)$ y con significancia estadística ( $p$ $\leq 0,05)$, se obtuvieron entre el porcentaje de sólidos solubles totales y el peso del fruto $(r=$ $-0,72)$, en cuyo caso se obtuvo la regresión lineal $\left(R^{2}=0,52\right)$; y entre el porcentaje de sólidos solubles totales y la edad al inicio de la cosecha $\left(r=-0,71 ; R^{2}=0,50\right)$. Se obtuvieron otras 10 correlaciones altas y con significancia estadística, según el tipo de tomate o el momento de siembra.

\section{Keywords}

Solanum lycopersicum; quality; yield; 'Brix; Pearson correlation; linear regression.

\begin{abstract}
For 63 tomato genotypes grown under greenhouse conditions the researchers estimated Pearson correlations among seven quantitative variables: number of days to the start of harvest (days after transplant), fruit weight (g), number of fruits per cluster, yield (ton/ha), fruit firmness (N), percentage of total soluble solids ( ${ }^{\circ} \mathrm{Brix}$ ), and fruit juice $\mathrm{pH}$. The only two Pearson correlations evaluated in the 63 genotypes that were high $(r \geq 0,69)$ and statistically significant $(p \leq 0,05)$, were obtained among percentage of total soluble solids and fruit weight $(r=-0,72)$, showing a linear regression statistic $\left(R^{2}\right)$ of 0.52 ; and among percentage of total soluble solids and the number of days to the start of harvest $\left(r=-0,71 ; R^{2}=0,50\right)$;. Statistically significant correlations were observed in ten additional cases, not across all genotypes but estimated according the type of tomato or the planting date.
\end{abstract}

\section{Introducción}

En el caso del tomate producido bajo invernadero, se cultivan principalmente genotipos de crecimiento indeterminado, de diferentes tamaños de fruto: pequeños ('cherry' y uva), medianos ('cocktail'), y grandes (gordos, para racimo, y 'saladette') [1].

Cada genotipo de tomate presenta diferentes características en cuanto al ciclo del cultivo, calidad del fruto, tolerancia a plagas y enfermedades, respuesta a las condiciones ambientales, entre otros. Es deseable que un cultivar presente buen rendimiento, resistencia a enfermedades, buena calidad del fruto, adaptabilidad a las condiciones ambientales donde se pretende cultivar, un mercado aceptable, y una larga vida de anaquel.

La calidad de los frutos de tomate está definida por sus características físicas (como el tamaño y la firmeza) y químicas (como pH y porcentaje de sólidos solubles totales), y por su calidad nutricional [1]. 
En esta hortaliza, el sabor está determinado por la concentración de azúcares tales como fructosa y glucosa, y de ácidos orgánicos como ácido cítrico y ácido málico [2], [3], [4], [5], [6]. En cuanto al aroma, hay más de 400 compuestos volátiles que contribuyen al mismo. La influencia del genotipo sobre estas características es muy importante [2], [3], [4], [7], [6].

Se ha determinado que los tomates tipo 'uva' tienen la misma intensidad de sabor que otros tomates, pero son mucho más dulces debido a que tienen un contenido de azúcares de casi 10 -Brix, característica que los hace un producto saludable y atractivo que ha tenido mucho éxito de ventas, y que ha incentivado su consumo en hogares y restaurantes [8].

Se presenta una gran variación en la respuesta de un genotipo bajo ambientes protegidos distintos, debido a diferencias en altitud, ubicación, grado de tecnificación, condiciones ambientales, entre otros. Por lo tanto, es aconsejable realizar ensayos con diferentes genotipos en cada invernadero, para escoger el que mejor se comporta en esas condiciones, y de acuerdo al mercado de destino de la producción [1]. En varios países se han realizado trabajos en esta dirección [9], [10], [11], [12], [13], [14], [15], [16], [6], [17], [18], [19], [20], [21], [22], [23], [24], [25], [26], [27].

El tipo de tomate juega un papel importante en el resultado de las correlaciones entre variables en este cultivo. En Francia, unos investigadores no encontraron correlaciones significativas para 18 híbridos de tomate gordo, entre las variables peso del fruto, firmeza del fruto, porcentaje de sólidos solubles totales, y $\mathrm{pH}$ [3]; sin embargo, para 17 híbridos de tomate 'cocktail' evaluados en dicho estudio, sí se encontraron correlaciones significativas entre el peso del fruto y la firmeza del fruto; entre el peso del fruto y el porcentaje de sólidos solubles totales; y entre el $\mathrm{pH}$ y el porcentaje de sólidos solubles totales; mientras que en ese caso, las correlaciones no fueron significativas entre el $\mathrm{pH}$ y el peso del fruto; entre el $\mathrm{pH}$ y la firmeza del fruto; ni entre la firmeza del fruto y el porcentaje de sólidos solubles totales.

El objetivo de esta investigación fue establecer correlaciones de Pearson entre siete variables cuantitativas para el cultivo de tomate bajo ambiente protegido, en Alajuela, Costa Rica.

\section{Materiales y métodos}

Se sembraron 63 genotipos de tomate (Solanum lycopersicum L.), correspondientes a seis tipos diferentes: 'cherry', 'cocktail', gordo, marmande, uva, y en forma de pera (cuadro 1).

La siembra se realizó en condiciones hidropónicas en el invernadero de Hortalizas de la Estación Experimental Agrícola Fabio Baudrit Moreno (EEAFBM), la cual está localizada en Barrio San José de Alajuela, Costa Rica, a una altitud de 883 msnm

Debido a la gran cantidad de genotipos por evaluar, se realizaron dos momentos de siembra diferentes. El primer grupo de genotipos $(n=38)$ se trasplantó el 4 de setiembre de 2012, y el segundo grupo de genotipos $(n=42)$ se trasplantó los días 25 de setiembre, 2 de octubre y 9 de octubre de 2012, según el desarrollo de las plántulas de cada híbrido.

Para el primer grupo de genotipos, la cosecha inició el día 16 de noviembre de 2012, es decir a los 73 días después de trasplante (ddt), y la evaluación de los frutos se llevó a cabo hasta el 8 de enero de 2013 (126 ddt). Para el segundo grupo de genotipos, la cosecha inició el día 16 de noviembre de 2012 (52 ddt), y la evaluación de los frutos se llevó a cabo hasta el 22 de enero de 2013 (105 ddt, 112 ddt y 119 ddt, según cada genotipo). Es importante indicar que, para ambos grupos, la producción se extendió más allá del período evaluado, pero no fue posible cuantificar dicha producción adicional.

El cultivo se realizó en sacos de fibra de coco, de $1 \mathrm{~m}$ de largo, $20 \mathrm{~cm}$ de ancho y $15 \mathrm{~cm}$ de altura. La distancia de siembra fue de $25 \mathrm{~cm}$ entre plantas, y de 1,54 m entre hileras, para 
una densidad de 2,60 plantas $/ \mathrm{m}^{2}$. Las plantas se sujetaron por medio de dos mallas plásticas, ubicadas una a cada lado de cada hilera de plantas. Todas las plantas se manejaron a un solo tallo, eliminando todos los tallos secundarios.

Cuadro 1. Genotipos de tomate utilizados en la investigación.

\begin{tabular}{|c|c|}
\hline Tipo de tomate & Genotipos \\
\hline $\begin{array}{l}\text { 'Cherry' } \\
(n=25)\end{array}$ & $\begin{array}{l}\text { Tangerino (United Genetics); } 8263 \text { (Nirit); Amarillo (DP Seeds); Mini Charm (DP Seeds); Mini } \\
\text { Star (DP Seeds); Cherry } 1 \text { (Green Seeds); Cherry } 2 \text { (Green Seeds); Cherry } 3 \text { (Green Seeds); } \\
\text { Cherry } 4 \text { (Green Seeds); Baby Cakes (DP Seeds); Grande Charm (DP Seeds); Sweet Cherry } \\
\text { (Western Hybrid); BHN-624 (Johnny's); BHN-968 (Johnny's); Black Cherry (Johnny's); Favorita } \\
\text { (Johnny's); Gold Nugget (Johnny's); Matt's Wild Cherry (Johnny's); Sakura (Johnny's); Sun } \\
\text { Cherry (Johnny's); Sun Gold (Johnny's); Tomatoberry Garden (Johnny's); Washington Cherry } \\
\text { (Johnny's); White Cherry (Johnny's); Yellow Mini (Johnny's) }\end{array}$ \\
\hline $\begin{array}{l}\text { 'Cocktail' } \\
(n=3)\end{array}$ & 72919 (Nirit); Giovanni (Nirit); Z-484 (Nirit) \\
\hline $\begin{array}{l}\text { Gordo } \\
(n=17)\end{array}$ & $\begin{array}{l}\text { Endeavour (Rijk Zwaan); 68-39-179 (Pandia); 68-39-177 (Pandia); VT-4 (Namdhari); } \\
\text { Furia (Green Seeds); Aida (Nirit); Metropol (Nirit); Otelo (Nirit); Tatiana (Nirit); } \\
7026 \text { (Nirit); NP-498 (Tan Nong Phat); IT-01-27 (DP Seeds); Pamella (DP Seeds); } \\
\text { Sacramento (DP Seeds); NS-537 (Namdhari); Tropic (Green Seeds); Criollo (Rijk } \\
\text { Zwaan) }\end{array}$ \\
\hline Marmande $(n=2)$ & Grandma's Little Girl (DP Seeds); Grandma's Pick (DP Seeds) \\
\hline $\begin{array}{l}\text { Forma de pera } \\
\qquad(n=1)\end{array}$ & Yellow Pear (Johnny's) \\
\hline $\begin{array}{c}\text { Uva } \\
(n=15)\end{array}$ & $\begin{array}{l}\text { Grappolino (Nirit); Lunico (Nirit); Hy Brix (DP Seeds); Pink Beauty (DP Seeds); Red } \\
\text { Grape (DP Seeds); Uva Roja (DP Seeds); Zuchello (DP Seeds); Dolce Vita (DP } \\
\text { Seeds); Red Scorpion (DP Seeds); Sweet Grape (Western Hybrid); Five Star Grape } \\
\text { (Johnny's); Golden Sweet (Johnny's); Red Pearl (Johnny's); Sakura Honey (Johnny's); } \\
\text { Smarty (Johnny's) }\end{array}$ \\
\hline
\end{tabular}

Nota: Elaboración propia, a partir de los genotipos utilizados. El nombre entre paréntesis corresponde a la empresa productora de la semilla de cada genotipo.

A partir de los descriptores internacionales para el cultivo de tomate [28], se seleccionaron las siguientes variables a evaluar:

1. Edad al inicio de la cosecha (ddt): se obtuvo al registrar el día de inicio de la cosecha en cada parcela, según la fecha de trasplante.

2. Número de frutos por racimo: se obtuvo al seleccionar 10 racimos por parcela, y se contó el número de frutos por racimo, y se calculó el promedio.

3. Peso del fruto (g): se midió el peso individual de 20 frutos por cada parcela, y se calculó el promedio.

4. Rendimiento (ton/ha): se estimó el peso de los frutos de tomate producidos en una hectárea, a partir del rendimiento por planta y la densidad de siembra. El rendimiento se evaluó hasta los 126 ddt en la primera prueba, y entre los 105 y 119 ddt en la segunda prueba.

5. Firmeza del fruto (N): se midió la firmeza de 20 frutos por cada parcela, y se obtuvo el promedio.

6. Porcentaje de sólidos solubles totales ( ${ }^{\circ}$ Brix): se midió el porcentaje de sólidos solubles totales de 20 frutos por cada parcela, y se calculó el promedio. 
7. $\mathrm{pH}$ del jugo del fruto: se evaluó el pH del jugo de 10 frutos por cada parcela, y se obtuvo el promedio.

8. Prueba de degustación: con 25 genotipos (13 'cherry'; 9 uva; 2 gordo; y 1 'cocktail'), se realizó una prueba de degustación (también llamado análisis sensorial [29], [8]), entre 61 personas, en la cual se realizó una evaluación cuantitativa de esta característica, para lo cual se empleó la siguiente escala hedonística de cinco puntos: 0 = pésimo; 1 = malo; 2 = regular; 3 = bueno; 4 = muy bueno. Para cada tipo de tomate se obtuvo un promedio de las evaluaciones recibidas.

El peso de los frutos se obtuvo con una balanza electrónica marca Ocony, modelo TH-I-EK, de $5000,0 \pm 0,1 \mathrm{~g}$ de capacidad. El porcentaje de sólidos solubles totales se determinó con un refractómetro manual marca Atago, modelo $\mathrm{N}-1$ a, con una escala de 0,0 - 32,0 \pm 0,2 ${ }^{\circ} \mathrm{Brix}$. Para la evaluación de firmeza del fruto, se utilizó un penetrómetro marca Chatillon, modelo DPP-100N, con una capacidad de $100 \pm 1 \mathrm{~N}$. Para la evaluación del pH se utilizó un medidor electrónico de pH marca Hanna Instruments, modelo HI 98129, con escala de 0,00 - 14,00 \pm 0,01.

Para cada genotipo se sembró una parcela con 8 plantas (2 sacos), y todos los datos se obtuvieron a partir de los frutos totales producidos en dicha parcela.

Para obtener la comparación entre los dos momentos de siembra evaluados (primera y segunda siembra), se utilizó la prueba de t de Student con una significancia de $5 \%$ para determinar diferencias entre tratamientos, donde los tratamientos fueron cada momento de siembra, y cada genotipo correspondió a una repetición; esta comparación se realizó únicamente entre los 17 genotipos que fueron sembrados en ambos momentos de siembra.

Además, para las siete variables evaluadas, se obtuvo el coeficiente de correlación de Pearson ( $r$ ) entre ellas, y para aquellas combinaciones de variables en que se obtuvo una alta correlación $(r \geq 0,69)$ y significancia estadística $(p \leq 0,05)$, se obtuvo la regresión lineal con su respectiva ecuación y coeficiente de determinación $\left(R^{2}\right)$. Las correlaciones se obtuvieron tanto entre el total de los 63 genotipos evaluados, como entre cada uno de los dos momentos de siembra, y así como según el tipo de tomate (excepto para los tipos Marmande y Pera, en cuyo caso el número de repeticiones fue muy bajo).

\section{Resultados y discusión}

No se presentaron diferencias estadísticamente significativas para la firmeza del fruto, el peso del fruto ni el rendimiento, entre la primera y la segunda siembra (cuadro 2). Sin embargo, sí se presentaron diferencias altamente significativas $(p \leq 0,01)$ entre ambos momentos de siembra para la edad al inicio de la cosecha, el pH, y el porcentaje de sólidos solubles totales; en la segunda siembra las plantas empezaron a producir frutos en forma más precoz (seis días antes que en la primera siembra), y los frutos presentaron un mayor $\mathrm{pH}$ y un mayor porcentaje de sólidos solubles totales, en comparación a los resultados obtenidos en la primera siembra.

Se obtuvo una alta correlación de Pearson $\left(r=0,71 ; p \leq 0,01 ; R^{2}=0,504\right)$ entre el porcentaje de sólidos solubles totales y la prueba de degustación (no se muestran los datos). En otro ensayo, también se había determinado una importante correlación de Pearson $\left(r=0,69 ; p \leq 0,01 ; R^{2}=\right.$ 0,476), entre ambas variables, al usar la misma escala hedonística de 0-4, entre varios genotipos de tomate producidos en invernadero [13]. En Estados Unidos, otros investigadores evaluaron seis genotipos de tomate en invernadero, y también encontraron una correlación positiva significativa entre el porcentaje de sólidos solubles totales y la evaluación de degustación (sabor) $\left(R^{2}=0,892\right)[30]$. 
En los cuadros 3 al 19, se presentan las correlaciones de Pearson para cada una de las combinaciones de las variables evaluadas. Solamente no se presentan los resultados para las combinaciones en que la correlación entre los 63 genotipos resultó ser no significativa; esto sucedió para las siguientes combinaciones: $\mathrm{pH}$ y peso del fruto; $\mathrm{pH}$ y rendimiento; $\mathrm{pH}$ y firmeza del fruto; y pH y número de frutos por racimo. Tampoco se presentan los resultados de las correlaciones por tipo de tomate, en los casos en que ninguna de ellas fue significativa, lo cual sucedió para las siguientes combinaciones: peso del fruto y edad a inicio de cosecha; porcentaje de sólidos solubles totales y rendimiento; porcentaje de sólidos solubles totales y firmeza; porcentaje de sólidos solubles totales y número de frutos por racimo; edad a inicio de cosecha y número de frutos por racimo; y rendimiento y edad a inicio de cosecha.

Con respecto a la relación entre $\mathrm{pH}$ y peso del fruto, en un ensayo en Francia, los tomates pequeños ('cherry') tuvieron un $\mathrm{pH}$ apenas ligeramente más alto $(4,26)$ en comparación con tomates medianos y grandes $(4,20-4,22)$ [3]. En el presente ensayo la correlación entre ambas variables para los 63 genotipos no fue estadísticamente significativa.

Cuadro 2. Influencia del momento de siembra sobre las variables evaluadas.

\begin{tabular}{|c|c|c|}
\hline & \multicolumn{2}{|c|}{ Momento de siembra } \\
\hline Variable & Primera siembra & Segunda siembra \\
\hline Edad al inicio de la cosecha (ddt) & $75,24 \mathrm{~b}$ & $37,60 \mathrm{a}$ \\
\hline Firmeza del fruto $(\mathrm{N})$ & $35,96 \mathrm{a}$ & $48,92 \mathrm{a}$ \\
\hline Peso del fruto $(\mathrm{g})$ & $58,73 \mathrm{a}$ & $4,09 \mathrm{~b}$ \\
\hline $\mathrm{pH}$ & $3,83 \mathrm{a}$ & $6,84 \mathrm{~b}$ \\
\hline Porcentaje de sólidos solubles totales ( ${ }^{\circ}$ Brix) & $5,24 \mathrm{a}$ \\
\hline Rendimiento (ton/ha) & $37,07 \mathrm{a}$ & $42,29 \mathrm{a}$ \\
\hline
\end{tabular}

Nota: valores con una letra en común entre tratamientos no son significativamente diferentes, según la prueba de t de Student $(p \leq 0,05)$. Esta comparación se estableció únicamente entre los 17 genotipos de tomate que se sembraron en ambos momentos de siembra.

Cuadro 3. Coeficientes de correlación de Pearson entre porcentaje de sólidos solubles totales ('Brix) y peso del fruto $(g)$.

\begin{tabular}{|c|c|c|c|}
\hline Genotipos & $\begin{array}{c}\text { Coeficiente de } \\
\text { correlación }(r)\end{array}$ & Probabilidad $(p)$ & $\begin{array}{c}\text { Coeficiente de determinación } \\
\text { de la regresión lineal }\left(R^{2}\right)\end{array}$ \\
\hline Total $(n=63)$ & $-0,72$ & ${ }^{* \star}$ & 0,52 \\
\hline Primera siembra $(n=38)$ & $-0,76$ & ${ }^{* \star}$ & 0,58 \\
\hline Segunda siembra $(n=42)$ & $-0,64$ & ${ }^{* *}$ & \\
\hline Cherry $(n=25)$ & $-0,58$ & ${ }^{* \star}$ & \\
\hline Cocktail $(n=3)$ & $-0,67$ & $n s$ & \\
\hline Gordo $(n=17)$ & $-0,29$ & $n s$ & \\
\hline Uva $(n=15)$ & $-0,43$ & $n s$ & \\
\hline
\end{tabular}

Nota: $n s=$ no significativo; ${ }^{*}=$ significativa $(\mathrm{p} \leq 0,05) ;{ }^{* \star}=$ altamente significativa $(\mathrm{p} \leq 0,01)$.

En el cuadro 3 se presentan las correlaciones de Pearson entre el porcentaje de sólidos solubles totales y el peso del fruto, y en la figura 1 se presenta la regresión lineal entre ambas variables $\left(R^{2}=0,52\right)$. Para los 63 genotipos, la correlación obtenida fue negativa y alta $(r=-0,72)$, y además fue altamente significativa, lo que indica que se obtiene una relación inversamente 
proporcional, pues conforme se reduce el peso del fruto, aumenta el porcentaje de sólidos solubles totales. Para los genotipos cultivados en la primera siembra, la correlación también fue alta $\left(r=-0,76 ; R^{2}=0,58\right)$, y altamente significativa.

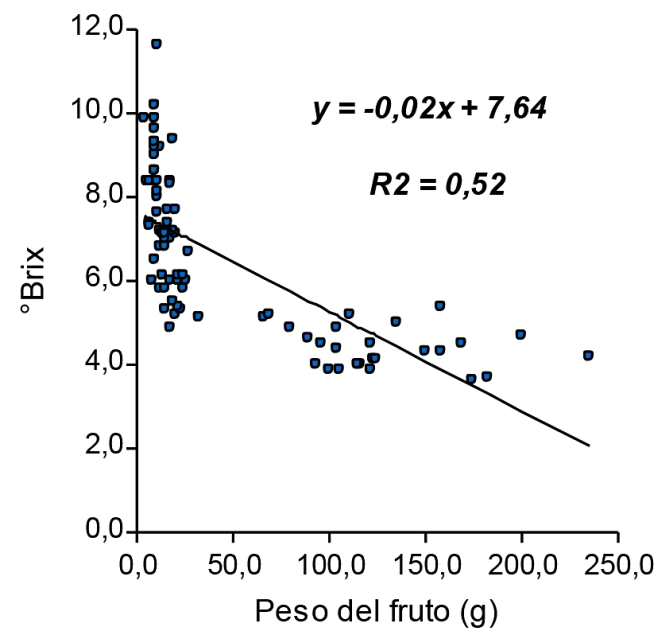

Figura 1. Regresión lineal del porcentaje de sólidos solubles totales ( ${ }^{\circ}$ Brix) versus el peso del fruto para el total de genotipos evaluados $(n=63)$.

Otros autores evaluaron 14 genotipos de tomate 'cocktail' y gordo en India, y encontraron una correlación alta, negativa y significativa entre el peso del fruto y el porcentaje de sólidos solubles totales $(r=-0,787)$ [31], lo cual es un resultado similar al obtenido en el presente trabajo. De la misma forma, en otra investigación realizada en Francia con 17 híbridos de tomate 'cocktail', se encontró también una correlación alta, negativa y significativa entre ambas variables $(r=-0,69)[3]$.

En el cuadro 4 se presentan los coeficientes de correlación de Pearson entre el porcentaje de sólidos solubles totales y la edad al inicio de la cosecha, y en la figura 2 se presenta la regresión lineal entre ambas variables $\left(R^{2}=0,50\right)$. Para los 63 genotipos se obtuvo una correlación negativa alta $(r=-0,71)$ y altamente significativa, es decir, que hay una relación inversamente proporcional entre ambas variables, pues conforme aumenta la edad a inicio de la cosecha se obtiene un menor porcentaje de sólidos solubles totales. También se obtuvo una correlación alta y significativa $\left(r=-0,86 ; R^{2}=0,74\right)$ para los tomates tipo 'cocktail'.

Cuadro 4. Coeficientes de correlación de Pearson entre porcentaje de sólidos solubles totales ( $\left.{ }^{\circ} \mathrm{Brix}\right)$ y edad al inicio de la cosecha (ddt).

\begin{tabular}{|c|c|c|c|}
\hline Genotipos & $\begin{array}{c}\text { Coeficiente de } \\
\text { correlación }(r)\end{array}$ & Probabilidad $(p)$ & $\begin{array}{c}\text { Coeficiente de determinación } \\
\text { de la regresión lineal }\left(\mathrm{R}^{2}\right)\end{array}$ \\
\hline Total $(n=63)$ & $-0,71$ & ${ }^{* \star}$ & 0,50 \\
\hline Primera siembra $(n=38)$ & $-0,52$ & ${ }^{* *}$ & \\
\hline Segunda siembra $(n=42)$ & $-0,50$ & ${ }^{* *}$ & \\
\hline Cherry $(n=25)$ & $-0,53$ & ${ }^{* \star}$ & 0,74 \\
\hline Cocktail $(n=3)$ & $-0,86$ & ${ }^{*}$ & \\
\hline Gordo $(n=17)$ & $-0,39$ & $n s$ & \\
\hline Uva $(n=15)$ & $-0,52$ & ${ }^{*}$ & \\
\hline
\end{tabular}

Nota: $n s=$ no significativo; ${ }^{*}=$ significativa $(p \leq 0,05) ;{ }^{* \star}=$ altamente significativa $(p \leq 0,01)$. 


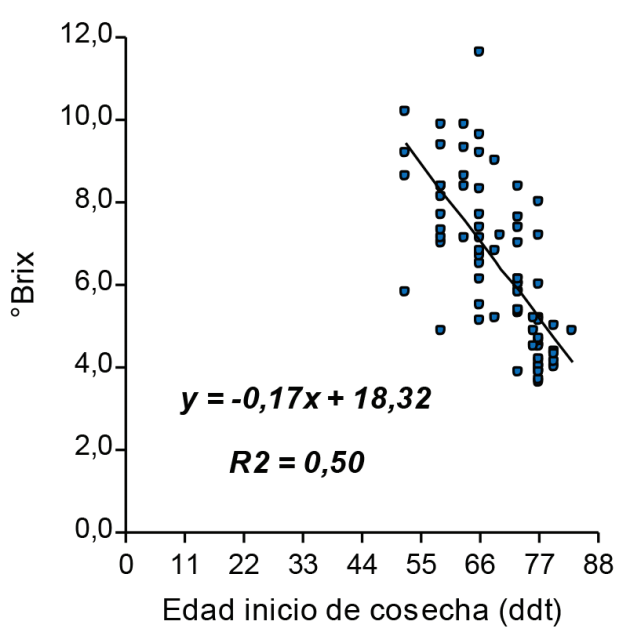

Figura 2. Regresión lineal del porcentaje de sólidos solubles totales ( $\left.{ }^{\circ} B r i x\right)$ versus la edad al inicio de la cosecha (ddt) para el total de genotipos evaluados $(n=63)$.

Con respecto a la relación entre el porcentaje de sólidos solubles totales y el rendimiento, en India unos autores evaluaron 14 genotipos de tomate 'cocktail' y gordo, y encontraron una correlación positiva y significativa entre ambas variables $(r=0,636)$ [31]. En forma contraria, en el presente trabajo las correlaciones significativas obtenidas fueron negativas en todos los casos ( $r=-0,56$ para el total de genotipos) (cuadro 5), y en el caso de los tomates tipo 'cocktail' y gordo sí fueron positivas, pero no fueron significativas (no se muestran los datos). En la figura 3 se presenta la regresión lineal en el caso del grupo de genotipos cultivados en la primera siembra $\left(r=-0,74 ; R^{2}=0,55\right)$; se obtuvo una relación inversamente proporcional, donde a mayor rendimiento se obtiene un menor porcentaje de sólidos solubles totales.

Cuadro 5. Coeficientes de correlación de Pearson entre porcentaje de sólidos solubles totales ('Brix) y rendimiento (ton/ha).

\begin{tabular}{|c|c|c|c|}
\hline Genotipos & $\begin{array}{c}\text { Coeficiente de } \\
\text { correlación }(r)\end{array}$ & Probabilidad $(p)$ & $\begin{array}{c}\text { Coeficiente de determinación de } \\
\text { la regresión lineal }\left(\mathrm{R}^{2}\right)\end{array}$ \\
\hline Total $(\mathrm{n}=63)$ & $-0,56$ & ${ }^{* \star}$ & 0,31 \\
\hline Primera siembra $(\mathrm{n}=38)$ & $-0,74$ & ${ }^{* \star}$ & 0,55 \\
\hline Segunda siembra $(\mathrm{n}=42)$ & $-0,48$ & ${ }^{* \star}$ & \\
\hline
\end{tabular}

Nota: $n s=$ no significativo; ${ }^{*}=$ significativa $(p \leq 0,05) ;{ }^{* *}=$ altamente significativa $(p \leq 0,01)$.

En el cuadro 6 se presentan los coeficientes de correlación de Pearson entre el porcentaje de sólidos solubles totales y el número de frutos por racimo, y en la figura 4 se presenta la regresión lineal para el grupo de genotipos cultivados en la primera siembra $\left(R^{2}=0,66\right)$, en cuyo caso se obtuvo una alta correlación positiva $(r=0,81)$, es decir, que a mayor número de frutos por racimo se obtiene un mayor porcentaje de sólidos solubles totales.

Con respecto a la relación entre peso y firmeza del fruto, en un ensayo desarrollado en Francia, se encontró que los tomates 'cherry' (13 g/fruto) presentaban una menor firmeza que los tomates más grandes (entre 129 y $151 \mathrm{~g} /$ fruto). Además, entre los tomates de frutos pequeños, la firmeza mostró una correlación positiva con el peso del fruto, pero esta correlación fue negativa en el caso de los tomates con frutos grandes [3]. En dicho estudio, para 17 híbridos de tomate 'cocktail' evaluados, se encontró una correlación alta, positiva y significativa entre el 
peso del fruto y la firmeza del fruto $(r=0,72)$ [3]. En el presente estudio se obtuvo un resultado similar al incluir los 63 genotipos de tomate $(r=0,67)$ (cuadro 7), y la correlación positiva fue todavía mayor en el grupo de genotipos evaluados en la primera siembra $(r=0,71)$, en cuyo caso se calculó la regresión lineal (figura 5) $\left(R^{2}=0,50\right)$. Estos resultados indican que la firmeza del fruto es mayor conforme aumenta el peso del fruto.

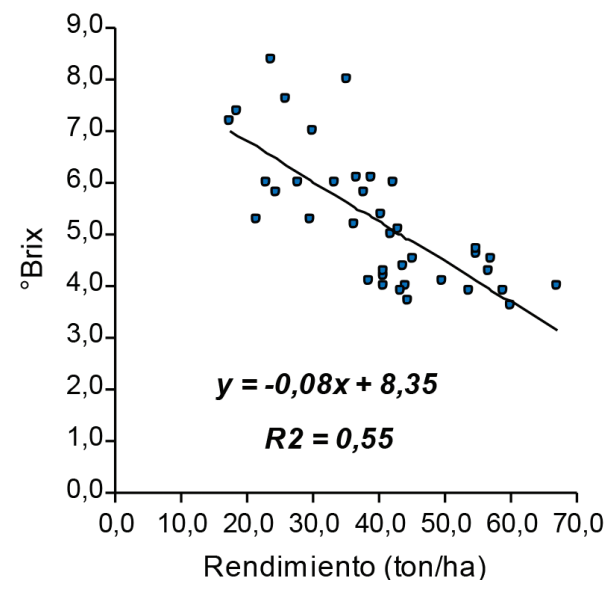

Figura 3. Regresión lineal del porcentaje de sólidos solubles totales ('Brix) versus el rendimiento (ton/ha) para los genotipos evaluados en la primera siembra $(n=38)$.

Cuadro 6. Coeficientes de correlación de Pearson entre porcentaje de sólidos solubles totales ('Brix) y número de frutos por racimo.

\begin{tabular}{|c|c|c|c|}
\hline Genotipos & $\begin{array}{c}\text { Coeficiente de } \\
\text { correlación }(r)\end{array}$ & Probabilidad $(p)$ & $\begin{array}{c}\text { Coeficiente de determinación de } \\
\text { la regresión lineal }\left(\mathrm{R}^{2}\right)\end{array}$ \\
\hline Total $(\mathrm{n}=63)$ & 0,61 & ${ }^{*}$ & 0,37 \\
\hline Primera siembra $(\mathrm{n}=38)$ & 0,81 & ${ }^{*}$ & 0,66 \\
\hline Segunda siembra $(\mathrm{n}=42)$ & 0,52 & ${ }^{*}$ & \\
\hline
\end{tabular}

Nota: $n s=$ no significativo; ${ }^{*}=$ significativa $(p \leq 0,05) ;{ }^{* \star}=$ altamente significativa $(p \leq 0,01)$.

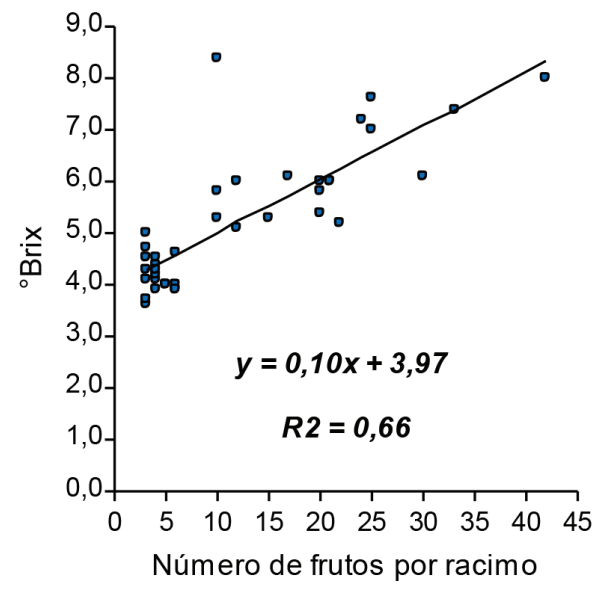

Figura 4. Regresión lineal del porcentaje de sólidos solubles totales ( ${ }^{\circ}$ Brix) versus el número de frutos por racimo para los genotipos evaluados en la primera siembra $(n=38)$. 
En el cuadro 8 se presentan los coeficientes de correlación de Pearson entre el rendimiento y la firmeza del fruto, y en la figura 6 se presenta la regresión lineal para los genotipos cultivados en la primera siembra $\left(R^{2}=0,48\right)$, en cuyo caso se obtuvo una correlación positiva alta $(r=0,69)$, y altamente significativa; es decir, que a mayor rendimiento se obtuvo una mayor firmeza del fruto.

Cuadro 7. Coeficientes de correlación de Pearson entre firmeza del fruto $(\mathrm{N})$ y peso del fruto $(\mathrm{g})$.

\begin{tabular}{|c|c|c|c|}
\hline Genotipos & $\begin{array}{c}\text { Coeficiente de } \\
\text { correlación }(r)\end{array}$ & Probabilidad $(p)$ & $\begin{array}{c}\text { Coeficiente de determinación de } \\
\text { la regresión lineal }\left(R^{2}\right)\end{array}$ \\
\hline Total $(n=63)$ & 0,67 & $* *$ & 0,45 \\
\hline Primera siembra $(n=38)$ & 0,71 & $* *$ & \\
\hline Segunda siembra $(n=42)$ & 0,54 & $*$ & \\
\hline Cherry $(n=25)$ & 0,40 & $n s$ & \\
\hline Cocktail $(n=3)$ & 0,45 & * & \\
\hline Gordo $(n=17)$ & 0,39 & 0,51 & \\
\hline Uva $(n=15)$ & & & \\
\hline
\end{tabular}

Nota: $n s=$ no significativo; ${ }^{*}=$ significativa $(p \leq 0,05) ;{ }^{* *}=$ altamente significativa $(p \leq 0,01)$.

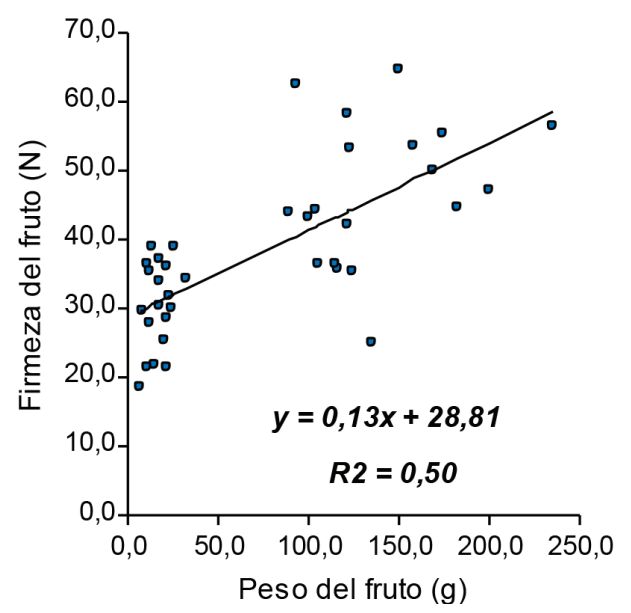

Figura 5. Regresión lineal de la firmeza del fruto versus el peso del fruto para los genotipos evaluados en la primera siembra $(n=38)$.

Cuadro 8. Coeficientes de correlación de Pearson entre rendimiento (ton/ha) y firmeza del fruto (N).

\begin{tabular}{|c|c|c|c|}
\hline Genotipos & $\begin{array}{c}\text { Coeficiente de } \\
\text { correlación }(r)\end{array}$ & Probabilidad $(p)$ & $\begin{array}{c}\text { Coeficiente de determinación } \\
\text { de la regresión lineal }\left(R^{2}\right)\end{array}$ \\
\hline Total $(n=63)$ & 0,54 & ${ }^{*}$ & 0,29 \\
\hline Primera siembra $(n=38)$ & 0,69 & ${ }^{*}$ & 0,48 \\
\hline Segunda siembra $(n=42)$ & 0,39 & ${ }^{*}$ & \\
\hline Cherry $(n=25)$ & 0,46 & ${ }^{* \star}$ & \\
\hline Cocktail $(n=3)$ & 0,07 & $n s$ & \\
\hline Gordo $(n=17)$ & 0,12 & $n s$ & \\
\hline Uva $(n=15)$ & 0,42 & $n s$ & \\
\hline
\end{tabular}

Nota: $n s=$ no significativo; ${ }^{*}=$ significativa $(p \leq 0,05) ;{ }^{* *}=$ altamente significativa $(p \leq 0,01)$. 


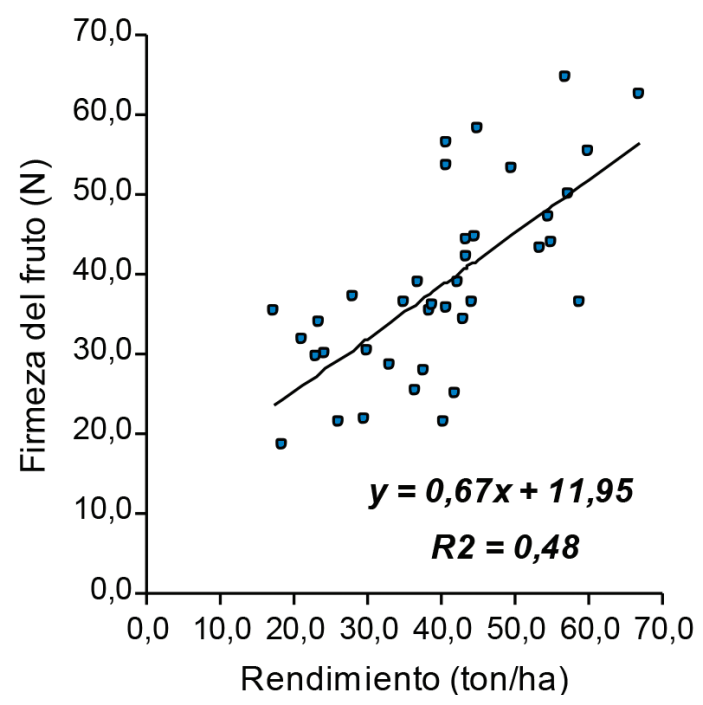

Figura 6. Regresión lineal de la firmeza del fruto (N) versus el rendimiento (ton/ha) para los genotipos evaluados en la primera siembra $(\mathrm{n}=38)$.

En el cuadro 9 se presentan los coeficientes de correlación de Pearson entre el número de frutos por racimo y el peso del fruto, y en la figura 7 se presenta la regresión lineal para el grupo de genotipos evaluados en la primera siembra $\left(R^{2}=0,61\right)$, en cuyo caso la correlación fue alta y negativa $(r=-0,78)$, y altamente significativa, lo que indica una relación inversamente proporcional, es decir, que a mayor número de frutos por racimo se obtiene un menor peso del fruto; esto se explica por la partición de fotoasimilados en un mayor número de órganos sumidero. En el caso de los tomates tipo gordo, también se obtuvo una correlación alta $(r=$ $\left.-0,70 ; R^{2}=0,49\right)$, y altamente significativa.

En el cuadro 10 se presentan los coeficientes de correlación de Pearson entre el rendimiento y el número de frutos por racimo, y en la figura 8 se presenta la regresión lineal para los genotipos tipo 'cocktail' ( $R^{2}=0,70$ ), en cuyo caso se obtuvo una correlación positiva y alta $(r=0,84)$, y estadísticamente significativa; es decir, que a mayor número de frutos por racimo se obtuvo un mayor rendimiento.

Cuadro 9. Coeficientes de correlación de Pearson entre número de frutos por racimo y peso del fruto (g).

\begin{tabular}{|c|c|c|c|}
\hline Genotipos & $\begin{array}{c}\text { Coeficiente de } \\
\text { correlación }(r)\end{array}$ & $\begin{array}{c}\text { Probabilidad }(p) \\
\text { Coefiente de determinación de } \\
\text { la regresión lineal }\left(R^{2}\right)\end{array}$ \\
\hline Total $(n=63)$ & $-0,67$ & $\star \star$ & 0,45 \\
\hline Primera siembra $(n=38)$ & $-0,78$ & $\star *$ & \\
\hline Segunda siembra $(n=42)$ & $-0,53$ & $n s$ & \\
\hline Cherry $(n=25)$ & 0,07 & $n s$ & 0,49 \\
\hline Cocktail $(n=3)$ & 0,79 & $\star \star$ & \\
\hline Gordo $(n=17)$ & $-0,70$ & $n s$ & \\
\hline Uva $(n=15)$ & $-0,45$ & $n$ \\
\hline
\end{tabular}

Nota: $n s=$ no significativo; ${ }^{*}=$ significativa $(p \leq 0,05) ;{ }^{* *}=$ altamente significativa $(p \leq 0,01)$. 


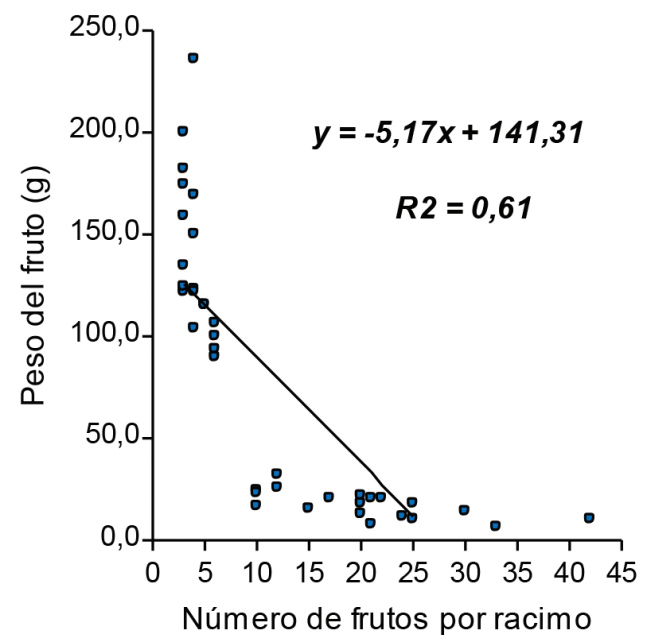

Figura 7. Regresión lineal del peso del fruto versus el número de frutos por racimo para los genotipos evaluados en la primera siembra $(n=38)$.

Cuadro 10. Coeficientes de correlación de Pearson entre rendimiento (ton/ha) y número de frutos por racimo.

\begin{tabular}{|c|c|c|c|}
\hline Genotipos & $\begin{array}{c}\text { Coeficiente de } \\
\text { correlación }(r)\end{array}$ & Probabilidad $(p)$ & $\begin{array}{c}\text { Coeficiente de determinación de } \\
\text { la regresión lineal }\left(R^{2}\right)\end{array}$ \\
\hline Total $(n=63)$ & $-0,42$ & $* *$ & 0,18 \\
\hline Primera siembra $(n=38)$ & $-0,61$ & ${ }^{* *}$ & \\
\hline Segunda siembra $(n=42)$ & $-0,23$ & $n s$ & 0,70 \\
\hline Cherry $(n=25)$ & 0,20 & $n s$ & \\
\hline Cocktail $(n=3)$ & 0,84 & ${ }^{*}$ & \\
\hline Gordo $(n=17)$ & 0,45 & ${ }^{* *}$ & \\
\hline Uva $(n=15)$ & $-0,06$ & $n s$ & \\
\hline
\end{tabular}

Nota: $n s=$ no significativo; ${ }^{*}=$ significativa $(p \leq 0,05) ;{ }^{* \star}=$ altamente significativa $(p \leq 0,01)$.

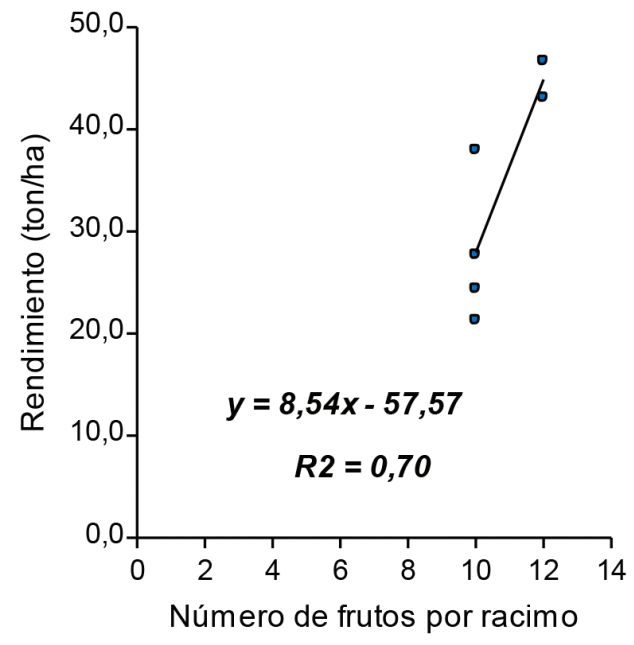

Figura 8. Regresión lineal del rendimiento (ton/ha) versus el número de frutos por racimo para los genotipos tipo 'cocktail' ( $n=3)$. 
En el cuadro 11 se presentan los coeficientes de correlación de Pearson entre el pH del jugo del fruto y la edad al inicio de la cosecha, y en la figura 9 se presenta la regresión lineal para los genotipos de tomate tipo 'cocktail' $\left(R^{2}=0,90\right)$; en este caso la correlación negativa obtenida fue alta $(r=-0,95)$, y altamente significativa, lo que indica una relación inversamente proporcional, en la que a mayor edad a inicio de cosecha se obtiene un menor $\mathrm{pH}$ del jugo de los frutos.

Cuadro 11. Coeficientes de correlación de Pearson entre pH del jugo del fruto y edad al inicio de la cosecha (ddt).

\begin{tabular}{|c|c|c|c|}
\hline Genotipos & $\begin{array}{c}\text { Coeficiente de } \\
\text { correlación }(r)\end{array}$ & Probabilidad $(p)$ & $\begin{array}{c}\text { Coeficiente de determinación de } \\
\text { la regresión lineal }\left(\mathrm{R}^{2}\right)\end{array}$ \\
\hline Total $(\mathrm{n}=63)$ & $-0,28$ & $* \star$ & 0,08 \\
\hline Primera siembra $(\mathrm{n}=38)$ & 0,13 & $\mathrm{~ns}$ & \\
\hline Segunda siembra $(n=42)$ & 0,22 & $n s$ & 0,90 \\
\hline Cherry $(n=25)$ & $-0,28$ & $n \mathrm{~ns}$ & \\
\hline Cocktail $(n=3)$ & $-0,95$ & $* \star$ & \\
\hline Gordo $(n=17)$ & $-0,27$ & $n s$ & \\
\hline Uva $(n=15)$ & $-0,63$ & $* *$ & \\
\hline
\end{tabular}

Nota: $n s=$ no significativo; ${ }^{*}=$ significativa $(p \leq 0,05) ;{ }^{* *}=$ altamente significativa $(p \leq 0,01)$.

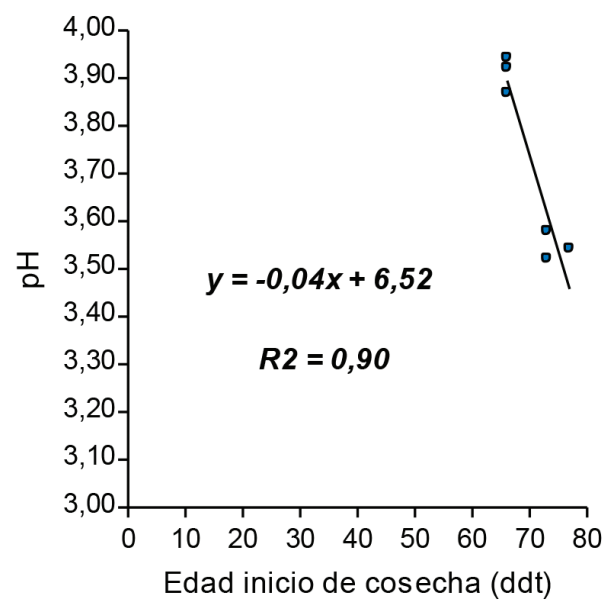

Figura 9. Regresión lineal del pH del jugo del fruto versus la edad al inicio de la cosecha (ddt) para los genotipos tipo 'cocktail' ( $n=3)$.

En India, unos autores evaluaron 14 genotipos de tomate 'cocktail' y gordo, y encontraron una correlación positiva y significativa entre el peso del fruto y el rendimiento $(r=0,345)$ [31]. Por otra parte, en un ensayo en México, se encontró entre 11 genotipos de tomate gordo, que la correlación entre el peso promedio del fruto y el rendimiento fue positiva e importante $(r=0,500)$, pero no fue estadísticamente significativa [10]. En comparación con estos resultados, en el presente trabajo dicha correlación positiva, al considerar el total de genotipos evaluados, fue un poco superior ( $r$ $=0,59$ ) pero no se consideró alta, aunque sí fue altamente significativa (cuadro 12).

En el cuadro 13 se presenta la correlación de Pearson entre la edad al inicio de la cosecha y el peso del fruto. La correlación fue positiva y altamente significativa, pero no se consideró alta $(r=0,62)$. 
Cuadro 12. Coeficientes de correlación de Pearson entre rendimiento (ton/ha) y peso del fruto (g).

\begin{tabular}{|c|c|c|c|}
\hline Genotipos & $\begin{array}{c}\text { Coeficiente de } \\
\text { correlación }(r)\end{array}$ & Probabilidad $(p)$ & $\begin{array}{c}\text { Coeficiente de determinación de } \\
\text { la regresión lineal }\left(\mathrm{R}^{2}\right)\end{array}$ \\
\hline Total $(\mathrm{n}=63)$ & 0,59 & ${ }^{* *}$ & 0,35 \\
\hline Primera siembra $(\mathrm{n}=38)$ & 0,67 & ${ }^{* *}$ & \\
\hline Segunda siembra $(\mathrm{n}=42)$ & 0,54 & ${ }^{* *}$ & \\
\hline Cherry $(\mathrm{n}=25)$ & 0,48 & ${ }^{* \star}$ & \\
\hline Cocktail $(\mathrm{n}=3)$ & 0,37 & $\mathrm{~ns}$ & \\
\hline Gordo $(\mathrm{n}=17)$ & $-0,24$ & $\mathrm{~ns}$ & \\
\hline Uva $(n=15)$ & 0,48 & ${ }^{*}$ & \\
\hline
\end{tabular}

Nota: $n s=$ no significativo; ${ }^{*}=$ significativa $(p \leq 0,05) ;{ }^{* \star}=$ altamente significativa $(p \leq 0,01)$.

Cuadro 13. Coeficientes de correlación de Pearson entre edad al inicio de la cosecha (ddt) y peso del fruto (g).

\begin{tabular}{|c|c|c|c|}
\hline Genotipos & $\begin{array}{c}\text { Coeficiente de } \\
\text { correlación }(r)\end{array}$ & Probabilidad $(p)$ & $\begin{array}{c}\text { Coeficiente de determinación de } \\
\text { la regresión lineal }\left(R^{2}\right)\end{array}$ \\
\hline Total $(n=63)$ & 0,62 & ${ }^{* *}$ & 0,38 \\
\hline Primera siembra $(n=38)$ & 0,65 & ${ }^{* *}$ & \\
\hline Segunda siembra $(n=42)$ & 0,66 & ${ }^{* *}$ & \\
\hline
\end{tabular}

Nota: $n s=$ no significativo; ${ }^{*}=$ significativa $(p \leq 0,05) ;{ }^{* *}=$ altamente significativa $(p \leq 0,01)$.

En un estudio en España, se evaluaron 19 genotipos de tomate, y se encontró una correlación estadísticamente no significativa entre el $\mathrm{pH}$ y el porcentaje de sólidos solubles totales $(r=$ $0,184)[5]$. En la presente investigación, la correlación positiva entre ambas variables fue un poco superior ( $r=0,28)$, aunque todavía muy baja, y además fue altamente significativa (cuadro 14).

Cuadro 14. Coeficientes de correlación de Pearson entre porcentaje de sólidos solubles totales ( $\left.{ }^{\circ} \mathrm{Brix}\right)$ y pH del jugo del fruto.

\begin{tabular}{|c|c|c|c|}
\hline Genotipos & $\begin{array}{c}\text { Coeficiente de } \\
\text { correlación }(r)\end{array}$ & Probabilidad $(p)$ & $\begin{array}{c}\text { Coeficiente de determinación de } \\
\text { la regresión lineal }\left(R^{2}\right)\end{array}$ \\
\hline Total $(n=63)$ & 0,28 & ${ }^{* *}$ & 0,08 \\
\hline Primera siembra $(n=38)$ & $-0,02$ & $n s$ & \\
\hline Segunda siembra $(n=42)$ & $-0,03$ & $n s$ & \\
\hline Cherry $(n=25)$ & 0,39 & ${ }^{*}$ & \\
\hline Cocktail $(n=3)$ & 0,80 & $n s$ & \\
\hline Gordo $(n=17)$ & 0,63 & ${ }^{*}$ & \\
\hline Uva $(n=15)$ & 0,32 & $n s$ & \\
\hline
\end{tabular}

Nota: $n s=$ no significativo; ${ }^{*}=$ significativa $(p \leq 0,05) ;{ }^{* *}=$ altamente significativa $(p \leq 0,01)$.

En otra investigación en Francia, para 17 híbridos de tomate 'cocktail' evaluados, se encontró una correlación positiva y significativa entre el $\mathrm{pH}$ y el porcentaje de sólidos solubles totales ( $r$ $=0,68)[3]$, lo cual es un resultado superior al obtenido en el presente ensayo al contemplar los 63 genotipos, pero es similar al valor obtenido por los tomates gordos $(r=0,63)$. 
En el cuadro 15 se presentan los coeficientes de correlación de Pearson entre el porcentaje de sólidos solubles totales y la firmeza del fruto; la correlación negativa obtenida para los 63 genotipos fue altamente significativa, pero no se consideró alta $(r=-0,50)$.

Cuadro 15. Coeficientes de correlación de Pearson entre porcentaje de sólidos solubles totales ( ${ }^{\circ}$ Brix) y firmeza del fruto $(\mathrm{N})$.

\begin{tabular}{|c|c|c|c|}
\hline Genotipos & $\begin{array}{c}\text { Coeficiente de } \\
\text { correlación }(r)\end{array}$ & Probabilidad $(p)$ & $\begin{array}{c}\text { Coeficiente de determinación de } \\
\text { la regresión lineal }\left(R^{2}\right)\end{array}$ \\
\hline Total $(n=63)$ & $-0,50$ & $\star \star$ & 0,25 \\
\hline Primera siembra $(n=38)$ & $-0,57$ & $n s$ & \\
\hline Segunda siembra $(n=42)$ & $-0,26$ & $n s$ & \\
\hline
\end{tabular}

Nota: $n s=$ no significativo; ${ }^{*}=$ significativa $(p \leq 0,05) ;{ }^{* *}=$ altamente significativa $(p \leq 0,01)$.

En los cuadros 16 y 17 se presentan los coeficientes de correlación de Pearson entre la firmeza del fruto y las siguientes variables, respectivamente: edad al inicio de la cosecha; y número de frutos por racimo; en ambos casos, las correlaciones obtenidas para los 63 genotipos no fueron consideradas altas ( $r=0,61$ y $-0,46$, respectivamente), aunque sí fueron altamente significativas.

Cuadro 16. Coeficientes de correlación de Pearson entre firmeza del fruto (N) y edad al inicio de la cosecha (ddt).

\begin{tabular}{|c|c|c|c|}
\hline Genotipos & $\begin{array}{c}\text { Coeficiente de } \\
\text { correlación }(r)\end{array}$ & $\begin{array}{c}\text { Coeficiente de determinación de } \\
\text { la regresión lineal }\left(R^{2}\right)\end{array}$ \\
\hline Total $(n=63)$ & 0,61 & $\star \star$ & 0,37 \\
\hline Primera siembra $(n=38)$ & 0,49 & $\star *$ & \\
\hline Segunda siembra $(n=42)$ & 0,60 & $\star \star$ & \\
\hline Cherry $(n=25)$ & 0,51 & $n s$ & \\
\hline Cocktail $(n=3)$ & $-0,16$ & $n s$ & \\
\hline Gordo $(n=17)$ & 0,06 & $n s$ & \\
\hline Uva $(n=15)$ & 0,15 & $n$ & \\
\hline
\end{tabular}

Nota: $\mathrm{ns}=$ no significativo; ${ }^{*}=$ significativa $(\mathrm{p} \leq 0,05) ;{ }^{\star \star}=$ altamente significativa $(\mathrm{p} \leq 0,01)$.

Cuadro 17. Coeficientes de correlación de Pearson entre firmeza del fruto (N) y número de frutos por racimo.

\begin{tabular}{|c|c|c|c|}
\hline Genotipos & $\begin{array}{c}\text { Coeficiente de } \\
\text { correlación }(r)\end{array}$ & Probabilidad $(p)$ & $\begin{array}{c}\text { Coeficiente de determinación de } \\
\text { la regresión lineal }\left(R^{2}\right)\end{array}$ \\
\hline Total $(n=63)$ & $-0,46$ & $\star \star$ & 0,21 \\
\hline Primera siembra $(n=38)$ & $-0,59$ & $n s$ & \\
\hline Segunda siembra $(n=42)$ & $-0,28$ & $n s$ & \\
\hline Cherry $(n=25)$ & 0,27 & $n s$ & \\
\hline Cocktail $(n=3)$ & 0,22 & $n s$ & \\
\hline Gordo $(n=17)$ & $-0,35$ & $* \star$ & \\
\hline Uva $(n=15)$ & $-0,57$ & $n$ & \\
\hline
\end{tabular}

Nota: $\mathrm{ns}=$ no significativo; ${ }^{*}=$ significativa $(\mathrm{p} \leq 0,05) ;{ }^{\star \star}=$ altamente significativa $(\mathrm{p} \leq 0,01)$. 
En el cuadro 18 se presentan los coeficientes de correlación de Pearson entre la edad al inicio de la cosecha y el número de frutos por racimo; la correlación obtenida para los 63 genotipos no fue considerada alta $(r=-0,35)$, a pesar de ser altamente significativa.

Cuadro 18. Coeficientes de correlación de Pearson entre edad al inicio de la cosecha (ddt) y número de frutos por racimo.

\begin{tabular}{|c|c|c|c|}
\hline Genotipos & $\begin{array}{c}\text { Coeficiente de } \\
\text { correlación }(r)\end{array}$ & Probabilidad $(p)$ & $\begin{array}{c}\text { Coeficiente de determinación de } \\
\text { la regresión lineal }\left(R^{2}\right)\end{array}$ \\
\hline Total $(n=63)$ & $-0,35$ & $* *$ & 0,12 \\
\hline Primera siembra $(n=38)$ & $-0,52$ & $* *$ & \\
\hline Segunda siembra $(n=42)$ & $-0,32$ & $*$ & \\
\hline
\end{tabular}

Nota: $n s=$ no significativo; ${ }^{*}=$ significativa $(p \leq 0,05) ;{ }^{* \star}=$ altamente significativa $(p \leq 0,01)$.

En el cuadro 19 se presentan los coeficientes de correlación de Pearson entre el rendimiento y la edad al inicio de la cosecha. La correlación obtenida para los 63 genotipos fue altamente significativa, pero no fue considerada alta $(r=0,40)$.

Cuadro 19. Coeficientes de correlación de Pearson entre rendimiento (ton/ha) y edad al inicio de la cosecha (ddt).

\begin{tabular}{|c|c|c|c|}
\hline Genotipos & $\begin{array}{c}\text { Coeficiente de } \\
\text { correlación }(r)\end{array}$ & Probabilidad $(p)$ & $\begin{array}{c}\text { Coeficiente de determinación de } \\
\text { la regresión lineal }\left(R^{2}\right)\end{array}$ \\
\hline Total $(n=63)$ & 0,40 & $* *$ & 0,16 \\
\hline Primera siembra $(n=38)$ & 0,52 & $* \star$ & \\
\hline Segunda siembra $(n=42)$ & 0,41 & $* *$ & \\
\hline
\end{tabular}

Nota: $n s=$ no significativo; ${ }^{*}=$ significativa $(p \leq 0,05) ;{ }^{\star \star}=$ altamente significativa $(p \leq 0,01)$.

\section{Conclusiones}

Según las correlaciones de Pearson altas $(r \geq 0,69)$ y estadísticamente significativas $(p \leq 0,05)$ obtenidas, se concluye lo siguiente:

a) Para el grupo de 63 genotipos evaluados, se evidenció que a mayor peso del fruto y a mayor edad a inicio de cosecha, se obtuvo un menor porcentaje de sólidos solubles totales.

b) Para el grupo de 38 genotipos cultivados en la primera siembra, se evidenció que: la firmeza del fruto es mayor conforme aumenta el peso del fruto y el rendimiento; que el porcentaje de sólidos solubles totales es mayor conforme se reduce el rendimiento y conforme aumenta el número de frutos por racimo; y que el peso del fruto se redujo conforme aumenta el número de frutos por racimo.

c) Para el grupo de tres genotipos de tomate tipo 'cocktail', se evidenció que: el pH del jugo de los frutos se reduce conforme aumenta la edad a inicio de cosecha; y que el rendimiento se incrementa conforme aumenta el número de frutos por racimo. 


\section{Agradecimientos}

Los autores agradecen la colaboración de Jorge Díaz, Cristina Arguedas, Carolina Ramírez y Julio Vega en el trabajo de campo, y de Mario Monge en la revisión de la traducción del resumen al idioma inglés. Asimismo, agradecen el financiamiento recibido por parte de la Vicerrectoría de Investigación de la Universidad de Costa Rica, para la realización de este trabajo.

\section{Referencias}

[1] J. Z. Castellanos, Manual de producción de tomate en invernadero, Celaya, Guanajuato, México: Intagri S. C., 2009.

[2] A. Alonso, R. García-Aliaga, S. García-Martínez, J. J. Ruiz y A. A. Carbonell-Barrachina, «Characterization of spanish tomatoes using aroma composition and discriminant analysis., » Food Science and Technology International, vol. 15, $\mathrm{n}^{\circ}$ 1, pp. 47-55, 2009.

[3] M. Causse, M. Buret, K. Robini y P. Verschave, «Inheritance of nutritional and sensory quality traits in fresh market tomato and relation to consumer preferences, Journal of Food Science, vol. 68, n 7, pp. 2342-2350, 2003.

[4] J. Cebolla-Cornejo, S. Roselló, M. Valcárcel, E. Serrano, J. Beltrán y F. Nuez, «Evaluation of genotype and environment effects on taste and aroma flavor components of spanish fresh tomato varieties, 》 Journal of Agricultural and Food Chemistry, vol. 59, pp. 2440-2450, 2011.

[5] V. Fernández-Ruiz, M. C. Sánchez-Mata, M. Cámara, M. E. Torija, C. Chaya, L. Galiana-Balaguer, S. Roselló y F. Nuez, «Internal quality characterization of fresh tomato fruits, Hortscience, vol. 39, n 2, pp. 339-345, 2004.

[6] F. H. Shirahige, P. C. T. Melo, A. P. Jacomino, A. M. T. Melo, L. F. V. Purquerio y M. S. Roquejani, «Yield and qualitative characterization of fresh market tomato hybrids of Italian and Santa Cruz types, » Acta Horticulturae, vol. 821, pp. 81-88, 2009.

[7] D. Klein, V. Gkisakis, A. Krumbein, I. Livieratos y U. Köpke, «Old and endangered tomato cultivars under organic greenhouse production: effect of harvest time on flavour profile and consumer acceptance, » International Journal of Food Science and Technology, vol. 45, pp. 2250-2257, 2010.

[8] L. Pillsbury, E. Maynard y K. Hayes, «Chemical, physical and sensory properties of four grape tomato varieties, s.f.. [En línea]. Available: https://www2.ag.purdue.edu/hla/fruitveg/Documents/pdf/reports/grapetomato_in_04.pdf.

[9] G. Ardila, G. Fischer y H. E. Balaguera-López, «Caracterización del crecimiento del fruto y producción de tres híbridos de tomate (Solanum lycopersicum L.) en tiempo fisiológico bajo invernadero,» Revista Colombiana de Ciencias Hortícolas, vol. 5, n 1, pp. 44-56, 2011.

[10] F. Borrego, A. López, J. M. Fernández, M. Murillo, S. A. Rodríguez, A. Reyes y J. M. Martínez, «Evaluación agronómica de tomate (Lycopersicon esculentum M.) en invernadero, » Agronomía Mesoamericana, vol. 12, $\mathrm{n}^{\circ}$ 1, pp. 49-56, 2001.

[11] R. L. Grijalva-Contreras, R. Macías-Duarte y F. Robles-Contreras, «Comportamiento de híbridos de tomate bola en invernadero bajo condiciones desérticas del noroeste de Sonora,» Tropical and Subtropical Agroecosystems, vol. 14, pp. 675-682, 2011.

[12] J. E. Monge-Pérez, «Caracterización de 14 genotipos de tomate (Lycopersicon esculentum Mill.) cultivados bajo invernadero en Costa Rica, Tecnología en Marcha, vol. 27, nº 4, pp. 58-68, 2014.

[13] J. E. Monge-Pérez, «Evaluación de 60 genotipos de tomate (Lycopersicon esculentum Mill.) cultivados bajo invernadero en Costa Rica, Intersedes, vol. 16, n 33, pp. 84-122, 2015.

[14] J. E. Monge-Pérez, «Evaluación preliminar de 201 genotipos de ocho diferentes hortalizas (berenjena, chile dulce, zucchini, ayote, sandía, pepino, tomate y melón) cultivados bajo invernadero en Costa Rica, de La investigación en Guanacaste II, San José, Costa Rica, Nuevas Perspectivas, 2016, pp. 277-300.

[15] V. H. Montenegro, «Evaluación de la aclimatación de veinte y dos cultivares de tomate (Lycopersicon esculentum Mill), bajo invernadero, en Chugllin, cantón Chambo, Provincia Chimborazo,» Escuela de Ingeniería Agronómica, Facultad de Recursos Naturales, Escuela Superior Politécnica de Chimborazo, Riobamba, Ecuador, 2012.

[16] M. B. Pérez, M. Albarracín, H. Moratinos y F. Zapata, «Rendimiento y calidad de fruto en cuatro cultivares de tomate (Solanum lycopersicum L.) bajo condiciones protegidas, »Revista Facultad Agronomía (LUZ), vol. 29, pp. 395-412, 2012. 
[17] D. J. Cantliffe, N. L. Shaw, S. A. Sargent, C. Simms, A. Berry, E. L. Kan, L. Puentes y J. W. Scott, «The determinate 'Tasti-Lee' tomato competes with indeterminate, greenhouse cultivars for yield, fruit quality, and sensory analysis when produced hydroponically,» Proceedings of the Florida State Horticultural Society, vol. 122, pp. 275-280, 2009.

[18] R. L. Grijalva-Contreras, R. Macías-Duarte, S. A. Grijalva-Durón, F. Núñez-Ramírez y F. Robles-Contreras, «Productividad de cultivares de tomate cherry bajo condiciones de invernadero en el noroeste de Sonora,» Biotecnia, vol. 16, nº 2, pp. 27-30, 2014.

[19] R. L. Grijalva-Contreras, F. Robles-Contreras y R. Macías-Duarte, Híbridos de tomate para la producción en invernadero en el noroeste de Sonora, Hermosillo, Sonora, México: Instituto Nacional de Investigaciones Forestales, Agrícolas y Pecuarias, 2009, p. 34.

[20] H. d. J. Herrera, A. Hurtado-Salazar y N. Ceballos-Aguirre, «Estudio técnico y económico del tomate tipo cereza élite (Solanum lycopersicum L. var. cerasiforme) bajo condiciones semicontroladas," Revista Colombiana de Ciencias Hortícolas, vol. 9, n², pp. 290-300, 2015.

[21] M. M. Maboko y C. P. Du Plooy, «Effect of pruning on yield and quality of hydroponically grown cherry tomato (Lycopersicon esculentum),» South African Journal of Plant and Soil, vol. 25, n 3, pp. 178-181, 2008.

[22] M. M. Maboko, C. P. Du Plooy y S. Chiloane, "Yield of determinate tomato cultivars grown in a closed hydroponic system as affected by plant spacing,» Horticultura Brasileira, vol. 35, n² 2, pp. 258-264, 2017.

[23] P. Mazuela, L. Acuña, M. Álvarez y A. Fuentes, «Producción y calidad de un tomate cherry en dos tipos de invernadero en cultivo sin suelo,» Idesia, vol. 28, n², pp. 97-100, 2010.

[24] M. d. Q. Rocha, R. M. N. Peil y C. M. Cogo, «Rendimento do tomate cereja em função do cacho floral e da concentração de nutrientes em hidroponia,» Horticultura Brasileira, vol. 28, n 4, pp. 466-471, 2010.

[25] M. L. Sancho, «Características poscosecha de cinco genotipos de tomate (Lycopersicon esculentum Mill.), en una localidad de clima templado (Estados Unidos) comparado con una localidad de clima tropical,» Sede Regional de San Carlos, Instituto Tecnológico de Costa Rica, Santa Clara, San Carlos, Costa Rica, 2013.

[26] P. F. Vargas, L. S. Duarte, E. H. C. Silva, A. C. Zecchini, R. S. Soares y L. J. G. Godoy, «Performance of mini-tomato hybrids in different training systems with different number of stems, "Horticultura Brasileira, vol. 35, $n^{\circ} 3$, pp. 428-433, 2017.

[27] S. M. Zahedi, N. A. Ansari y S. A. Eftekhari, «Investigation of yield and adaptation of ten selected genotypes of tomato under subtropical climate conditions (Ahvaz), » Journal of Food, Agriculture \& Environment, vol. 10, no 1, pp. 782-786, 2012.

[28] IPGRI, Descriptores para el tomate (Lycopersicon spp.), Roma, Italia: International Plant Genetic Resources Institute, 1996, p. 47.

[29] M. Moricz, «Investigations to the fruit quality and consumer acceptance of Heirloom tomato varieties,» s.f.. [En línea]. Available: http://labmath.eu.

[30] M. M. Peet, C. D. Harlow y E. S. Larrea, «Fruit quality and yield in five small-fruited greenhouse tomato cultivars under high fertilization regime,» Acta Horticulturae, vol. 659, pp. 811-818, 2004.

[31] R. Kumar, N. K. Mishra, J. Singh, G. K. Rai, A. Verma y M. Rai, «Studies on yield and quality traits in tomato (Solanum lycopersicon (Mill.) Wettsd.), Vegetable Science, vol. 33, n² 2, pp. 126-132, 2006. 